\title{
Commentary: Impacts of intensive follow-up on the long-term prognosis of percutaneous coronary intervention in acute coronary syndrome patients - a single center prospective randomized controlled study in a Chinese population
}

\author{
Ping-Shuan Dong ${ }^{1 *}$, Jing-Jing Jia' ${ }^{1}$, Lai-Jing Du'
}

*1Department of Cardiology, The First Affiliated Hospital, and College of Clinical Medicine of Henan University of Science and Technology, Luoyang, China

\section{Article Info}

\section{Article Notes}

Received: February 14, 2018

Accepted: March 01, 2018

\section{*Correspondence:}

Dr. Ping-Shuan Dong, Department of Cardiology, The First Affiliated Hospital, and College of Clinical Medicine of Henan University of Science and Technology, Luoyang 471003 ,

China; Tel: 13608650819;

E-mail: pingshuandong@foxmail.com

(c) 2018 Dong PS. This article is distributed under the terms of the Creative Commons Attribution 4.0 International License.

\section{Key Words}

Coronary artery disease

Percutaneous coronary intervention

Disease management

Cardiovascular risk factor

Medication adherence

\begin{abstract}
Various forms of interventional programs have been suggested to help reduce the risk factors for cardiovascular diseases. However, little is known about the effects of physician-based interventional program on the long-term disease prognoses of patients after percutaneous coronary intervention (PCI). A number of studies have demonstrated the intensive secondary prevention provide effective risk factor reduction in coronary artery disease patients, while few studies focused on important cardiovascular outcomes and long-term clinical prognoses. This study is the first prospective randomized controlled study to demonstrate the impact of professional physician-coordinated intensive follow-up on the long-time prognosis of $\mathrm{PCl}$ in acute coronary syndrome (ACS) patients in China. We have shown that this intensive follow-up program robustly prolongs the survival time and reduces the controllable risk factors for cardiovascular disease control of cardiovascular risk factors, thereby markedly improve the prognosis of $\mathrm{PCl}$ in ACS patients. The study was the first to demonstrate the cost-effect benefit of intensive secondary prevention in ACS patients in Chinese populations, and this benefit was long-term.
\end{abstract}

The 2010 Global Burden of Disease study demonstrated that the burden of ischemic heart disease and stroke has risen significantly since the first similar study in 1990. These conditions were ranked number one and three respectively for global Disability Adjusted Life Years, and remain the top two causes of mortality in the world. Franco has pointed out that the age-old adage that "prevention is better than cure" has been extensively tested to determine which interventions are most effective and which are most cost effective. Numerous evidences have shown that lifestyle intervention and risk factor management not only reduce cardiovascular morbidity and mortality in patients with established atherosclerotic disease but also in high-risk people. Examples of telehealth interventions include telephone quit lines for cigarette smokers, telemonitoring devices for blood pressure in patients' homes, and interactive online food and exercise diaries or tailored mobile phone text messages for weight loss. Researchers have also begun assessing the effect of mobile devices in disease prevention.

A report from Chartbook on Trends in the Health of American $2007^{1}$ showed that the decrease of cardiovascular disease mortality, $44-67 \%$ was attributable to to the control of risk factors. 
In China, aging and population growth is estimated to increase cardiovascular disease by more than $50 \%$ in the coming 20 years. However, numerous studies have clearly demonstrated that these cardiovascular risk factors are poorly controlled and the medication compliance is low. More specifically, the risk factors control in patients after coronary revascularization is far beyond optimal. Various forms of interventional programs have been suggested to help reduce the risk factors for cardiovascular diseases. However, little is known about the effects of physician-based interventional program on the long-term disease prognoses of patients after percutaneous coronary intervention (PCI).

This study is the first prospective randomized controlled study to demonstrate the impact of professional physician-coordinated intensive follow-up on the longtime prognosis of PCI in acute coronary syndrome (ACS) patients in China. We have shown that this intensive follow-up program robustly prolongs the survival time and reduces the controllable risk factors for cardiovascular disease control of cardiovascular risk factors, thereby markedly improve the prognosis of PCI in ACS patients. The study was the first to demonstrate the cost-effect benefit of intensive secondary prevention in ACS patients in Chinese populations, and this benefit was long-term.

A number of studies have demonstrated the intensive secondary prevention provide effective risk factor reduction in coronary artery disease patients, while few studies focused on important cardiovascular outcomes and long-term clinical prognoses. Merriel et al2 showed there was insufficient evidence to determine the effectiveness of telehealth interventions can reducing overall cardiovascular disease risk. The weak evidence for a reduction in systolic blood pressure and total cholesterol, and no change in High-Density Lipoprotein cholesterol or smoking rates. However, Neubeck et $\mathrm{al}^{3}$ concluded in a systematic review that interventions by telephone calls can lead effects on risk factor reduction and secondary prevention in the general population through effectively reducing the patient's total cholesterol, systolic blood pressure and lipoproteins. Wood et $\mathrm{al}^{4}$ showed that nursecoordinated multidisciplinary, family-based cardiovascular disease prevention program led to some weight loss, a reduction in central obesity, good blood pressure control and blood cholesterol, and especially the improvement was significant in high-risk patients. Compared to this nurse-coordinated program, our intensive follow-up program is coordinated by profession physicians. This allows a close monitor of disease status and risk factor, and more importantly a timely and professional adjustment of medications. Through this follow-up, the important cardiovascular events and the medical costs of patients after PCI can be marked reduced, and these are associated with a more ideal control of cardiovascular risk factors. To achieve the effects of the model of preventive cardiology, we need to go beyond specialized cardiac rehabilitation services and provide local preventive cardiology programs, appropriately adapted to the medical, cultural, and economic setting of a country.

\section{References}

1. National Center for Health Statistics (US). Health, United States, 2007: With Chartbook on Trends in the Health of Americans. National Center for Health Statistics (US); Report No.: 2007-1232.

2. Merriel SW, Andrews V, Salisbury C. Telehealth interventions for primary prevention of cardiovascular disease: a systematic review and meta-analysis. Prev Med. 2014; 64: 88-95.

3. Neubeck L, Redfern J, Fernandez R, et al. Telehealth interventions for the secondary prevention of coronary heart disease: a systematic review. Eur J Cardiovasc Prev Rehabil. 2009; 16(3): 281-9.

4. Wood DA, Kotseva K, Connolly S, et al. Nurse-coordinated multidisciplinary, family-based cardiovascular disease prevention programme (EUROACTION) for patients with coronary heart disease and asymptomatic individuals at high risk of cardiovascular disease: a paired, cluster-randomised controlled trial. Lancet. 2008; 371(9629): 1999-2012 